\title{
A Review Paper on DevOps: Beginning and More To Know
}

\author{
Pratibha Jha \\ Scholar \\ ABES Institute of Technology \\ Ghaziabad, U.P, India
}

\author{
Rizwan Khan \\ Associate Professor \\ ABES Institute of Technology \\ Ghaziabad, U.P, India
}

\begin{abstract}
DevOps is one of the conceptual studies for integration of operational and development review for Infrastructure and information system. The modern develop companies face multiple challenges to meet the requirement to develop software system and maintain the software quality. DevOps is an approach that merges the traditional Software roles and works on a way to enhance communication to improve deployment frequency rate and maintain the quality of Software. Here we will see how DevOps actually was introduced as a set of rules, tools, and practices to create more efficient ways to deal with the upcoming and existing challenges related to the software development and maintain its purpose with its operational leads. DevOps is basically a model that combines practices and tools that help to elevate core values. We can say DevOps being an ideal amalgamation archetypal of current developed procedure and tools that exaggerate the beliefs of DevOps.
\end{abstract}

\section{General Terms}

DevOps, Continuous development, Continuous integration, Continuous deployment

\section{Keywords}

DevOps, Continuous development, Continuous integration, Continuous deployment,CAMS.

\section{INTRODUCTION}

The expression "DevOps" regularly alludes to the rising proficient development that backers a community-oriented working connection among improvement and IT activities, bringing about the quick stream of arranged work (i.e., high convey rates) while at the same time expanding the dependability, steadiness, flexibility, and security of the creative environment.

It might be accepted that "DevOps" joins advancement and operational duties. Be that as it may, DevOps isn't tied in with consolidating customary duties into a solitary group. Or maybe, DevOps is an arrangement of managing standards and technique which advances programming perfection all through its SDLC life cycle. Boundaries between programming conveyance disciplines are separated to give consistent changes quickening time to advertise while enhancing quality. DevOps is centered around the accompanying objectives:

- $\quad$ Build up a culture of coordinated effort, separating conventional storehouses and basic measures of accomplishment.

- Quicken time to advertise with client input.

- Keep up current quality while driving enhancements for the life of the product through the usage of DevOps.

- Consider experimentation - organize bomb quick.

- Mechanize conventional operational and improvement duties.

- Use stages to give turnkey situations.

\section{HOW DID DevOps CAME INTO PICTURE?}

All were focused on a central issue that was ending up more pervasive over a developing number and assortment of IT associations: How would we conquer any hindrance among improvement and activities for the advancement of the business? One of the early discussions was between Patrick Dubois and Andrew Shaffer, IT experts, at the Speed Gathering 2008.

The consequence of that communication was one of the principal groups talking about this issue-the Lithe Frameworks Organization Gathering. This people group and resulting discussions drove Dubois to make the principal Speed Meeting (held in Belgium in October 2008), which he named "DevOps Days." Accordingly, the DevOps name and development were born.

The DevOps people group was to a great extent underground until 2011, when experts at Gartner and Red Monk wound up intrigued by the point. Presently, undertaking organizations started to demonstrate developing enthusiasm for DevOps. As the DevOps people group extended, it produced the advancement of horde instruments-including Vagrant, Rundeck, Manikin, Culinary specialist, Juju, Logstash, and some more-intended to enable DevOps to achieve its objectives.

Because of the early age of the development, DevOps isn't completely settled and still values being inexactly characterized. Archiving DevOps hones has started, however, no reasonable documentation of the group has been merged into standard accepted procedures. This implies each operational group applies the DevOps works on as indicated by authoritative requirements. Doing as such enables individuals to serve a wide range of parts, yet can prompt disarray when endeavoring to execute a DevOps culture in a conventional operational condition.

\subsection{Who Is Taking Advantages Of Devops?}

DevOps was initially viewed as a procedure discovered just in huge organizations (e.g., Netflix, Google) and new businesses. Inside the DevOps people group, these sorts of associations are frequently alluded to as "cloud-locals," "conceived in the cloud," or "unicorns.".

Today, the usage of DevOps can be found in three fundamentally extraordinary gatherings of adopters, all tested by the quickly expanding pace of use discharges

\section{A. Day by day to month to month discharges}

Early specialists of light-footed programming improvement techniques who try to quicken conveyance and organization to 
coordinate the fast pace of programming creation. The run of the mill discharge pace in these associations ranges from every day to month to month. A commonplace objective of early specialists is to wipe out improvement work-in-advance postponements caused by testing and operational discharge hold up times.

\section{B. Different every day discharges}

Web-scale experts, who receive DevOps to accomplish numerous miniaturized scale discharges in a day for an application or run-time benefit. In these situations, the quick refresh and arrival of code are attached to new highlights that are basic to meeting consistently developing client desires and aggressive dangers, and in addition to the basic to constantly. Analyze and move forward.

\section{Organized discharges}

Conventional IT associations working in settled (and regularly directed) businesses, for example, back have organized discharge hones. Every one of the three gatherings share a shared objective: disposing of the time and asset limitations of utilization conveyance while guaranteeing the quality, soundness, and constant change of their discharges and the experience for their end clients. The essential contrasts between the gatherings are driven by the recurrence and size of use discharges, generally in light of the business and nature of the administration conveyed.

\section{DevOps CYCLE}

DEVOPS cycle consist of:

\section{A. Continuous Integration}

Continuous Integration is an improvement hone that expects engineers to incorporate code into a mutual storehouse a few times each day. Each check-in is at that point confirmed by a robotized assemble, enabling groups to identify issues right on time in the cycle.

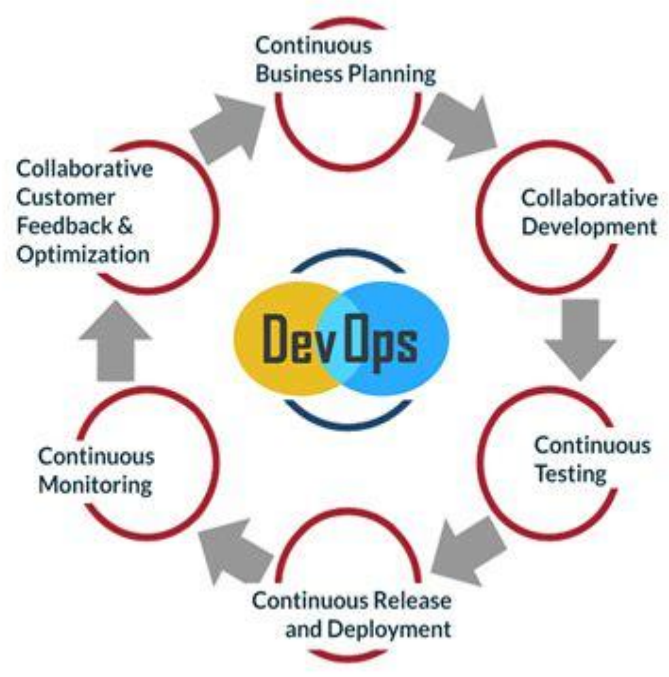

Figure 1:- The Devops Cycle

Consistent testing is the act of mechanizing and incorporating unit, useful, and non-useful (execution, security, and so on.) tests into the product conveyance chain and consequently executing those tests against each form of the code base. Particular tests and test conditions can be organized to guarantee the most applicable experiments are given need, or the whole test suite can be executed with each form. Nonstop testing can be used as a characteristic augmentation of test-driven advancement hones

\section{B. Continuous Delivery and Deployment}

Continuous conveyance is where you will likely form programming so it can be discharged to generation whenever, and it is dependable creation prepared. You accomplish nonstop conveyance via mechanizing the conveyance spill out of improvement to generation. Key components of persistent conveyance incorporate institutionalizing foundation setup, and overseeing arrangement subtle elements by following a similar teaching connected to overseeing source code.

\section{Continuous Operation}

Managing software and hardware changes in a way that is non-disruptive to end users. Processes such as patching and compliance fall under this function. Even though software and servers can be taken offline during planned maintenance, continuous operations enable customers to be serviced by the previous version of the application until the new version has been successfully tested and deployed.

\section{Continuous Assessment}

Continuously assessing an application, in view of three kinds of criticism-

1. Feedback circles-Persistently checking the accessibility, well being, and execution of the application, and additionally catching the client encounter all through the life cycle (i.e., improvement, quality confirmation, organizing, and generation) and bolstering it back to the fitting groups.

Doing as such empowers constant streamlining and adjusting of the application and end-client encounter.

2. Planning prioritization-As the arranging group gets input, they can persistently survey and organize new highlights, capacities, and deformity settling in view of business needs and end-client requests

3. Portfolio venture - As the arranging group gets input, they can constantly survey and organize speculations in light of business drivers.

Expected outcomes of DevOps:

- Velocity and speed of delivery to increase

- Quality and stability will improve

- Efficiency and capacity will increase.

\subsection{DevOps Core Values: CAMS}

CAMS which stands for culture, automation, measurement, and sharing. They impact the modern requirement of software development lifecycle.

A. Culture: Expel the storehouses among groups and upgrade interdepartmental correspondence. DevOps upgrade a source correspondence to enable all groups to impart and dispose of the impediments.

B. Automation: It spares time and furthermore averts defects, create consistency and empower the self-administration.

C. Measurement: DevOps help persistent conveyance and deployment. It underpins estimation and choices in view of obvious and simple to peruse information are the way to having right options.

D. Sharing: DevOps realized the power of sharing 
tools, findings, defects, and experiences enable the individual who shares similar interest

\subsection{What is DevOps not?}

\section{A. It's No Ops:}

It isn't "they're taking our employment!" A few people feel that DevOps implies that designers are assuming control tasks and doing it without anyone else 's help. Some portion of that is valid and part of it isn't.

It's a misguided judgment that DevOps is originating from the advancement side of the house to wipe out tasks - DevOps, and its forerunners in dexterous activities, are being started out of activities groups as a rule. This is on the grounds that activities people (and I represent myself here also) have understood that our current standards, procedures, and practices have not kept pace with what's required for progress. As organizations and improvement groups require greater spryness as the business atmosphere turns out to be all the more quick paced, we've frequently been giving less as we attempt to take care of our issues with greater unbending nature, and we require a central reorientation to have the capacity to give frameworks foundation in a powerful way.

Presently, as we understand a few sections of tasks should be mechanized, that implies that it is possible that we operations individuals do some computerization improvement, or engineers are stating "activities" code or both. That is startling to a few, however, is a piece of the estimation of the general community-oriented approach. All the fruitful groups I've run utilizing this approach have the two individuals with profound DEV ranges of abilities and profound operations ranges of abilities cooperating to make a superior general item. Also, I presently can't seem to see anybody mechanize themselves out of a vocation in cutting edge - as lower level concerns turn out to be more computerized, actually talented staff begin tackling the higher esteem issues up one level.

\section{B. It 's Not (Simply) Apparatuses:}

DevOps is additionally not just actualizing an arrangement of instruments. One motivation behind why I feel that an all the more usually acknowledged meaning of DevOps is required is that having different befuddling and inadequately organized definitions builds the hazard that individuals will go by the "hypothesis" and actualize the procedures or apparatuses of DevOps without the standards as a top priority, which is certainly an anti-pattern. Computerization is only the activity of energy, and impulsive

Robotization can do as much harm as savvy mechanization can bring advantage. Correspondingly, coordinated professionals would reveal to you that simply beginning to work in emphases or embracing other particular practices without starting important joint effort is probably going to not work out genuine well. There are a few groups of organizations I've worked for that received a portion of the techniques as well as apparatuses of dexterous however not its standards, and the outcomes were problematic. Without a doubt, an instrument can be helpful in Deft (or DevOps), yet in the event that you don't know how to utilize it at that point, it resembles giving an ambush weapon to an untrained individual.

However, at last, fussing about "devices shouldn't be called DevOps" is lost. Is poker arranging "coordinated" as in doing it mystically gets you Spry? No. In any case, it is a typical instrument utilized as a part of different nimble procedures, so calling it a "light-footed apparatus" is proper. Also, on the grounds that DevOps isn't only an entirety of the instruments doesn't imply that apparatuses particularly intended to run frameworks as per a DevOps attitude aren't significant.

\section{It's Not (Simply) Culture:}

Numerous individuals demand that DevOps "is simply culture" and you can't matter the word to a given rule or practice, however, I feel like this is exaggerated and mistaken. Coordinated has not helped a great many

DEV shops in light of the fact that the work on it ceased at "culture," with counsels to embrace colleagues and the lead specialists that distinguished the prescribed procedures basically pronouncing it was all plainly obvious and declining to be any more prescriptive. (In spite of the fact that there is some of that). DevOps comprises of things at all the levels I list above and is to a great extent futile without the unmistakable group of training that has developed around it. You may have the capacity to make sense of each one of those accepted procedures yourself given the social bearing and bunches of time to try, however sharing data is the reason we have the Web (and printing press besides).

\section{It's Not (Simply) DEV and Operations:}

What's more, at last, it's not exclusionary. A few people have griped "Shouldn't something be said about security individuals! Also, arrange administrators! Why abandon us out!?!" The fact of the matter is that every one of the members in making an item or framework ought to team up from the earliest starting point - business people of different stripes, engineers of different stripes, and activities people of different stripes, and this incorporates security, system, and whoever else. There are various sorts of business and engineer partners too; in light of the fact that everybody doesn't get a particular get out ("Bear in mind the symbol planners!") doesn't imply that they are excluded. The first light-footed advancement folks were, for the most part, contemplating "business + dev" $^{\text {c coordinated }}$ effort, and DevOps is bringing up issues and arrangements around "DEV + Operations" cooperation, however, the develop consequence of this is "everybody teaming up". In that sense, DevOps is only a noteworthy advance for one teacher to participate in the general culture of light-footed coordinated effort that ought to include all orders in an association. So whoever is taking an interest in the conveyance of the product or administration is a piece of DevOps.

\section{DevOps TOOLS PHASES}

DevOps is proposed to be a cross-useful method of working, instead of a solitary DevOps apparatus there are sets (or "toolchains") of numerous tools. Such DevOps devices are relied upon to fit into at least one of these classifications, intelligent of key parts of the improvement and conveyance process:

A) Code - code advancement and survey, source code administration devices, code combining

B) Construct - consistent joining instruments, fabricate status.

C) Test - constant testing apparatuses that give criticism on business dangers

D) Bundle - antiquity vault, application pre- arrangement organizing

E) Discharge - change administration, discharge endorsements, discharge robotization.

F) Design - framework setup and administration, Foundation as Code apparatuses

G) Screen - applications execution observing, end- client 


\section{encounter}

\subsection{Famous DevOps Tools Are}

\section{A). Continuous integration :}

When DevOps enthusiasts toss around phrases like "computerized framework," "foundation as code," and "programmable framework," they're discussing setup administration[3]. That is the following and controlling of changes to the product code base and the documenting of all record renditions into a focal arrangement administration database (CMDB), which empowers different designers to chip away at a similar code base while maintaining a strategic distance from variant control issues. Prevalent design administration apparatuses incorporate Ansible, CFEngine, Gourmet expert, Manikin, Rotten, SaltStack, and Ubuntu Juju. However, the genuine inquiry is what do you require in arrangement administration? For instance, in the event that you need to take in information that other individuals furnish and afterward accomplish something with it, you require an apparatus that handles that well.

\section{B) Application deployment:}

Application organization instruments empower the robotization of discharges and are at the core of consistent conveyance, one of the essential fundamentals of DevOps. Capistrano, an arrangement library, is the most well known independent device in this classification. Other well-known devices for robotizing application sending incorporate Ansible, Texture, and Jenkins. Once more, the key is to discover an instrument that tracks conduct-from history to change logs- in ways that is important for both dev and operations.

DevOps requires two particular sorts of checking. Application execution checking devices, for example, New Relic APM empower code-level recognizable proof and remediation of execution issues. At the framework level, server observing devices like New Relic Server give permeability into the limit, memory, and CPU utilization so unwavering quality architects can settle issues when they show up. The key is to ensure that everybody can see the information so they can settle on better choices.

\section{C) Monitoring-}

DevOps requires two particular sorts of checking. Application execution checking devices, for example, New Relic APM empower code-level recognizable proof and remediation of execution issues. At the framework level, server observing devices like New Relic Server give permeability into the limit, memory, and CPU utilization so unwavering quality architects can settle issues when they show up. The key is ensure that everybody can see the information so they can settle on better choices.

\section{D). Version control-}

To accomplish the advantages of DevOps, it's basic to the variant not only your application code but rather your framework, designs, and databases. This requires scripting the majority of your source curios, yet the result ought to be a solitary wellspring of truth for both your application code and your IT frameworks and databases, enabling you to rapidly recognize where things turned out badly, and reproduce known states with the push of a catch[5]. No all the more playing Sherlock Holmes to make sense of which variants of your application code runs with which conditions or databases. While normally utilized variant control apparatuses incorporate Git, Perforce, and Subversion, they contrast broadly on how well they bolster the DevOps-style joint effort.

\section{E). Test and construct frameworks.}

These apparatuses computerize basic designer undertakings including arranging source code into a paired code, making executable, running tests, and making documentation. Instruments in this classification incorporate Insect, Gradle, Jenkins, and Expert. Facilitated administrations like Travis and Build Hive offer extra alternatives.

The correct apparatus chain for DevOps will robotize IT administrations, give constant permeability into framework and application execution, and give you a solitary wellspring of truth. More imperative than an individual device's capacities, however, is the means by which intently the all match your association's key objectives. That is the best approach to expand your odds of accomplishing DevOps goodness.

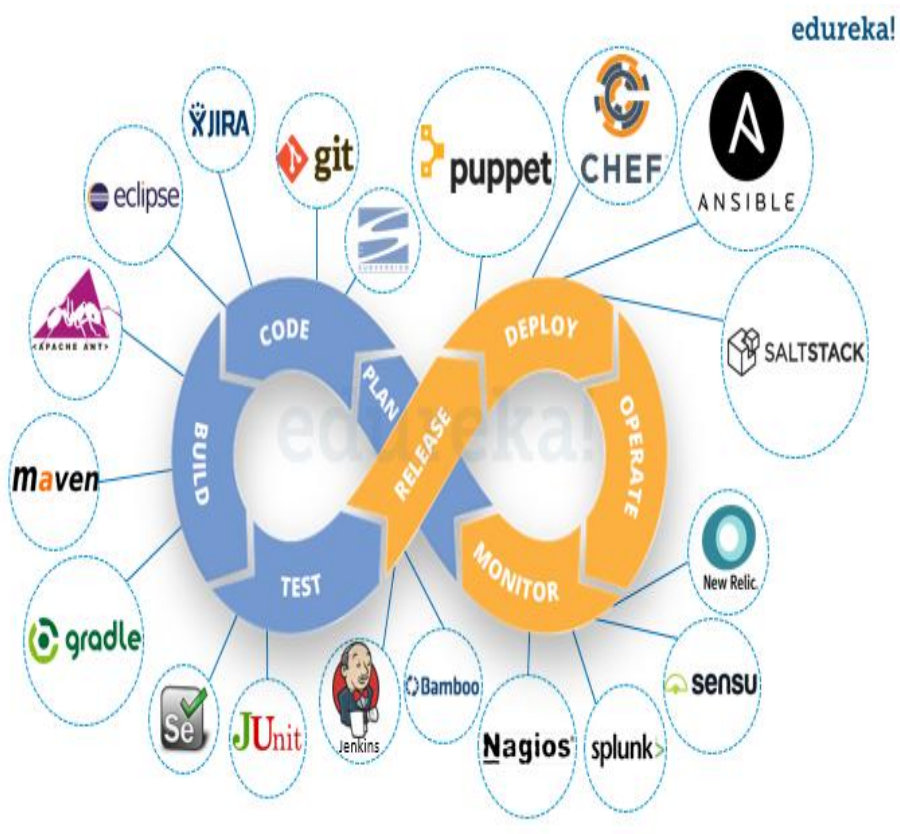

Figure 2: Devops Tools

\section{DEVELOPING DevOps ON AWS}

Actualizing DevOps in AWS is not kidding work, yet it won't be the overwhelming undertaking it appears. AWS bolsters DevOps endeavors by giving administrations to assemble, store and send applications. DevOps highlights are open in AWS, consistent reconciliation and constant conveyance boss among them

There are numerous apparatuses accessible in AWS that can help with DevOps, including Code Deploy, Code Pipeline, and Code Commit.[1] There is likewise Amazon Cloud Watch, which is basic for checking. Most associations should utilize a proof-of-idea utilization of the DevOps display and the innovation to show that it can be coordinated with existing advancement, testing, organization and activities forms. IT groups need to know whether DevOps is suitable for their associations, particularly in the event that they have applications in general society cloud.

Adequately utilizing DevOps enables a business to understand various effectiveness related advantages. On the off chance that your association is utilizing AWS and you need to exploit DevOps, it is imperative to know the ideal approaches to do as such. This handbook gives direction to IT masters searching for a place to begin and distinguishes a portion of some outsider devices accessible for assignments, for example, stack 
provisioning, test mechanization, design administration and framework observing.

\section{DevOps IN THE CLOUD}

Cloud-based processing guarantees, and frequently conveys, capacities, for example, versatile, virtualized endeavor arrangements, flexible foundations, strong administrations and develop stages Cloud-based engineering appears to offer boundless potential, however it likewise introduces numerous difficulties and dangers. Cloud-based registering is an extremely wide point that reaches from a private cloud inside an association's own foundation to leased administrations from a cloud supplier:

1. Software as a Service (SaaS): Programming gave on a membership or as-required premise. The specialist co-op deals with the hidden framework, including the working framework, and the application itself.

2. Platform as a Service (PaaS): The stage is kept up by the specialist organization to such an extent that the client has no worry for the hidden foundation, including the working framework and capacity.

3. Infrastructure as a Service (IaaS): The framework, including the boundless utilization of virtual private servers (VPS), is overseen by the supplier, with the end goal that the client keeps up the working framework, stockpiling, and foundation over the equipment and systems administration layer.

DevOps standards and practices give the foundational

capacities that make it workable for associations to make the best utilization of cloud-based processing and to address and relieve the innate dangers related to registering in the cloud]. Associations that can dependably fabricate their framework, arrangement servers, and convey applications are better situated to deal with the difficulties in the cloud. DevOps gives the accompanying center abilities that can help oversee cloud-based figuring:
a. Provisioning servers
b. Automated application deployment
c. Knowledge sharing
d. Managing the full ALM
e. Continuous integration and delivery
f. Continuous QA and testing
g. Infrastructure as code

Any product or frameworks advancement exertion must begin with a reasonable detail of the product and frameworks that are to be constructed. In the event that you can consequently construct your foundation, arrangement servers, and convey applications, your association can manage occurrences and issues that happen and consistently change specialist co-ops.

\section{DEVOPS IN FACEBOOK}

Facebook initially launched features like timeline, music, and ticker. Billions of people were on the server on the day of worldwide deployment. But due to the heavy traffic server got down. It was then Facebook launched "THE DARK TECHNIQUE" which was initially based on the feature of DevOps like continuous development and continuous testing, continuous monitoring and continuous deployment.

Firstly the small product is released and feedback is taken from the customer until the bug is not recovered.

\section{CONCLUSION}

In this review paper, we described, in short, the introduction, evolution, types, and components of DevOps.The examination demonstrates a few advantages and difficulties associated with embracing DevOps the advantages incorporate more continuous discharges, enhanced test mechanization, better correspondence and improved word related welfare. DevOps may be addressed for specific spaces and industry part, at any rate for the time being. the amusing meaning of DevOps additionally keeps organizations from having clear questions. This audit paper would comprehend the impacts in extensive scale, getting to the genuine estimation of DevOps.

\section{ACKNOWLEDGMENT}

We are thankful to Head of Department Mr. Dilkeshwar Pandey and all the faculty member of the Computer Science and engineering department of ABES Institute of Technology for motivation and encouragement which helped us to complete this review paper.

\section{REFERENCES}

[1] David Chapman, "Introduction to DevOps on AWS" in Amazon Web Services, December 2014, pp.no 1-20.

[2] S.W.Ambler. "Disciplined agile delivery and collaborative DevOps" in Cutter IT Journal 24.12(2011), pp. no.- $18-23$.

[3] SAUGATUK TECHNOLOGY, "Why DevOps Matters: practical insight on managing complex\& continuous change" in Microsoft, October 2014 Pages 1-8.

[4] Mohamed Khaled Aljundi, "Tools and Practices to Enhance DevOps Core Values”, 2016 pages 32-45.

[5] Rico de Feijter "Towards the adoption of DevOps in software product organization: A maturity model approach",May 23 ,2017, pp. 36-51"

[6] Leah Riungu-Kallosaari, "DevOps BENEFITS AND CHALLENGES IN PRACTICE”, NOV 2016 